\title{
Irritationen des professionellen Habitus?!
}

\section{Erste Einblicke in eine explorative Untersuchung der Handlungspraxis von Lehrkräften in der Inklusiven Erwachsenenbildung}

\section{Helge Stobrawe}

Journal für Psychologie, 27(2), 336-355

https://doi.org/10.30820/0942-2285-2019-2-336

www.journal-fuer-psychologie.de

\section{Zusammenfassung}

In diesem Beitrag werden Aufbau und erste relevante Befunde einer qualitativen Untersuchung zum professionellen Habitus der Lehrkräfte in der Erwachsenenbildung präsentiert. Ausgangspunkt ist der durch die Behindertenrechtskonvention (BRK) geforderte Wandlungsprozess der Umsetzung einer Inklusiven Erwachsenenbildung. Im Zentrum der Untersuchung steht die Rolle des professionellen Habitus, den dieser im Wandlungsprozess einnimmt. Unter Inklusiver Erwachsenenbildung wird das gemeinsame und gleichberechtigte Lernen von behinderten und nichtbehinderten Teilnehmenden verstanden und bezieht sich in diesem Fall auf Alphabetisierung und Zweiten Bildungsweg. Die übergeordnete Forschungsmethodik ist das Vorgehen nach der Grounded Theory. Das Ziel ist, ein besseres Verständnis für die mit dem professionellen Habitus verbundenen Mechanismen im Wandlungsprozess der Erwachsenenbildung zu entwickeln. Die Untersuchung ist ein Teilprojekt, das als Dissertationsvorhaben im Forschungsprojekt »Inklusive Bildung in der Alphabetisierungspraxis und im System des Zweiten Bildungswegs - Qualifikationen, Kompetenzen und Bedarfe des pädagogischen Personals « (INAZ) angesiedelt ist.

Schlüsselwörter: Behindertenrechtskonvention (BRK), Wandel, professioneller Habitus, Erwachsenenbildung, Behinderung

\section{Summary}

Irritations of the professional habitus?! First insights into an exploratory study concerning the practice of action of teachers in inclusive adult education

This paper presents the structure and first relevant findings of a qualitative study on the professional habitus of teachers in adult education. The starting point for this study is the process of change concerning the implementation of inclusive adult education promoted by the Convention on the Rights of Persons with Disabilities (CRPD). Therefore, the study focuses on 
the role the professional habitus has in this process of change. Inclusive adult education is understood as the teaching of disabled and non-disabled students without discrimination and on an equal basis, in this case referring to Literacy and Second Chance Education. The research methodology is the grounded theory approach. The aim is to develop a better understanding of the mechanisms associated with the professional habitus, in relation to the process of change in adult education. The study is located in the research project $\gg$ Inklusive Bildung in der Alphabetisierungspraxis und im System des Zweiten Bildungswegs - Qualifikationen, Kompetenzen und Bedarfe des pädagogischen Personals « (INAZ) as a $\mathrm{PhD}$ project.

Key words: Convention on the Rights of Persons with Disabilities (CRPD), change, professional habitus, adult education, disability

\section{$1 \quad$ Einleitung und Hintergrund}

Die Umsetzung der Behindertenrechtskonvention (BRK) in einfaches deutsches Recht am 26. März 2009 bildet den Ausgangspunktpunkt der im Folgenden präsentierten Forschungsarbeit. Verbunden mit der BRK wiederum ist das Verständnis von Inklusion, auf einer gemeinsamen rechtlichen Basis, gesellschaftliche Teilhabe für alle Menschen, in allen Lebensbereichen zu ermöglichen (Wansing 2015, 52). Diese rechtliche Verpflichtung durch die BRK begründet unter anderem den Anspruch behinderter Menschen auf gleichberechtigte Teilhabe im Bildungssystem nach dem Grundsatz der Nicht-Diskriminierung und Partizipation (Hirschberg 2010, 22ff.).

Der Fokus wissenschaftlicher Betrachtung lag und liegt dabei zumeist auf dem Schulsystem. Eher vernachlässigt wurde dagegen der Bildungsbereich der Erwachsenenbildung (Ackermann 2017, 134; Babilon 2018, 32; Meisel 2012, 20). Dies ist umso verwunderlicher, da die Erwachsenenbildung all jenen eine zweite Chance ermöglichen kann, die durch das Raster einer noch nicht oder immer noch nicht vorhandenen inklusiven Schulbildung gefallen sind.

Besondere Bedeutung kommt der Alphabetisierung und dem Zweiten Bildungsweg zu. Literalität stellt eine essenziell wichtige Kulturtechnik dar, die ebenso wie zertifizierte Bildungsabschlüsse, über die Möglichkeiten der gesellschaftlichen Partizipation entscheiden (Huinink und Schröder 2014, 110f.). Angesichts der Tatsache, dass die große Mehrheit von 71\% der Förderschüler*innen die Förderschule ohne Abschluss verlässt (BMAS 2016, 93), besitzt die Erwachsenenbildung das Potenzial, einen wichtigen Beitrag zu einem inklusiven Bildungssystem im Sinne der BRK zu leisten und durch Bildungsteilhabe, Teilhabe in anderen gesellschaftlichen Bereichen zu ermöglichen.

Ausdrücklich ist daher die Erwachsenenbildung Teil des rechtlichen Anspruchs auf inklusive Bildung (Art. 24 Abs. 5 BRK; Hirschberg und Lindmeier 2013, 42ff.). 
Deutschland ist als Vertragsstaat verpflichtet, den Zugang zu Erwachsenenbildung für behinderte Menschen ohne Diskriminierung und gleichberechtigt mit anderen sicherzustellen (Art. 24 Abs. 5 BRK). Erwachsenenbildungseinrichtungen sind daher gefordert, in ihren Angeboten dieser Verpflichtung zu begegnen (Hirschberg und Lindmeier 2013, 43ff.). Betroffen von dieser Forderung sind im besonderen Maße die Lehrkräfte in der Erwachsenenbildung. In ihrer beruflichen Praxis müssen diese schließlich die theoretisch normativen und rechtlich verbindlichen Vorgaben der BRK umsetzen.

Vermutlich existieren im Bereich von Alphabetisierung und Zweitem Bildungsweg sogar vielfältige Erfahrungen in dieser Hinsicht. Im Widerspruch hierzu lässt sich der Volkshochschulstatistik von 2018 (Reichart et al. 2018, 21f.) aber entnehmen, dass sich im Jahr 2017 lediglich 1,8\% der Kurse für spezielle Adressatengruppen an behinderte Menschen richteten. Hiervon entfielen lediglich 23,3\% auf die Grundbildung und nachholende Schulabschlüsse (ebd. 2018, 57). Es bleibt jedoch unklar, ob es sich um Kurse mit einem inklusiven Bildungsangebot handelte. Trotzdem lässt sich vermuten, dass durchaus mehr Kurse existieren, in denen behinderte und nichtbehinderten Menschen gemeinsam lernen. Diesen Schluss legen die Ergebnisse der wbmonitorUmfrage von 2012 nahe. Darin wird berichtet, dass 37\% aller Einrichtungen in der Weiterbildung angeben, dass behinderte Menschen am allgemeinen Kursangebot teilnehmen (Koscheck et al. 2013, 7). Lehrkräfte solcher Kursangebote können daher als Praxisexpert*innen angesehen werden, die über Erfahrungen hinsichtlich der für die Implementierung von für Inklusion notwendigen Handlungspraktiken verfügen könnten.

Konkreter Gegenstand dieses Beitrags ist daher die Handlungspraxis von Lehrkräften, die behinderte und nichtbehinderte Teilnehmende gemeinsam unterrichten. Unter Inklusiver Erwachsenenbildung wird dabei das gemeinsame und gleichberechtigte Lernen der Teilnehmenden verstanden und bezieht sich in diesem Fall auf die Alphabetisierung und den Zweiten Bildungsweg. Hierbei stellt sich die Frage, inwiefern die Lehrkräfte inklusive Bildung mit behinderten und nichtbehinderten Kursteilnehmenden umsetzen.

Die Forschungsarbeit ist ein eigenständiges Teilprojekt, das als Dissertationsvorhaben im Projekt »Inklusive Bildung in der Alphabetisierungspraxis und im System des Zweiten Bildungswegs - Qualifikationen, Kompetenzen und Bedarfe des pädagogischen Personals (INAZ) « angesiedelt ist, dessen Ziel die Entwicklung eines Fortbildungsmoduls für eine Inklusive Erwachsenenbildung ist. Der vorliegende Beitrag soll in das Dissertationsvorhaben und die zugrunde liegende Forschungsstrategie einführen, sowie einen ersten Einblick in die bereits gewonnenen Daten aus drei Gruppendiskussionen mit Lehrkräften aus der Erwachsenenbildung geben. Der vorliegende Beitrag soll in das Dissertationsvorhaben und die zugrunde liegende Forschungsstrate- 
gie einführen sowie einen ersten Einblick in die bereits gewonnenen Daten aus drei Gruppendiskussionen geben.

\section{Forschungsstrategie und theoretisch-methodisches Vorgehen}

Die übergeordnete Forschungsstrategie bildet das Vorgehen nach der Grounded Theory. Dies geschieht in der Tradition von Juliet Corbin und Anselm Strauss (2015) in Anlehnung an die konstruktivistische Weiterentwicklung von Kathy Charmaz (2014). Im Mittelpunkt steht das explorative Vorgehen bzw. das Erfassen und Verstehen der Logik von Handlungen und deren Begründung durch die Lehrkräfte (Charmaz 2014, 3; Corbin und Strauss 2015 11). Ziel des Vorgehens ist es, eine gegenstandbezogene Theorie zu entwickeln, welche die Handlungspraxis der Lehrkräfte in gemeinsamen Lernsettings von behinderten und nichtbehinderten Teilnehmenden verständlich nachvollziehbar macht.

Im Rahmen des konstruktivistischen Ansatzes der Grounded Theory wird davon ausgegangen, dass die entwickelte Theorie nicht ausschließlich aus den Daten resultiert. Im Prozess der Untersuchung bringen die Forscher*innen unweigerlich ihre eigene Sicht der Dinge in die Entwicklung der Theorie ein (Charmaz 2014, 12ff.; Corbin und Strauss 2015, 77f.). Die Herausforderung besteht hierbei darin, zu verhindern, dass das Forschungsergebnis durch die Forscher*innen unbemerkt in eine bestimmte Richtung gelenkt wird. Eine Möglichkeit ist, die Vorannahmen und Präkonzepte im Rahmen eines Sensitizing Concept (Charmaz 2014, 30f.) transparent und für den Forschungsprozess nutzbar zu machen.

Das Sensitizing Concept dient dann als Abgrenzung des Forschungsrahmens, bildet den Startpunkt der Untersuchung (ebd. 30f.) und kann abschließend zu den Ergebnissen ins Verhältnis gesetzt werden. Bei der Analysearbeit allerdings gilt es, stets die eigene Perspektive, im Hinblick auf den Einfluss auf den Forschungsprozess und dessen Ergebnisse, zu reflektieren (Corbin und Strauss 2015, 27). Dies bedeutet dann natürlich auch, den zuvor getroffenen Annahmen widersprechende Erkenntnisse zuzulassen.

\section{Theoretischer Ausgangspunkt (Sensitizing Concept)}

Als Sensitizing Concept dient hier das Habitus- und Feldkonzept Pierre Bourdieus (2005), sowie die subjektwissenschaftliche Lerntheorie nach Klaus Holzkamp (1995). Der Perspektive Helmut Bremers und Jana Trumanns (2013) folgend, werden die hier im Mittelpunkt stehenden Lehrkräfte als aktive und sozial eingebundene Individuen verstanden. Eingebunden sind die Lehrkräfte dabei in das deutsche Bildungssystem und 
seine Begründungslogiken, an dem diese ihre Handlungspraxis ausrichten. Eingebunden sind die Lehrkräfte aber auch in den durch die BRK vorangetriebenen Wandel, der Handlungsprobleme verursachen kann, denen diese aktiv begegnen müssen. Im Rahmen der explorativen Untersuchung dienen beide theoretischen Konzepte als Analysemodelle für die konkrete Handlungspraxis der Lehrkräfte. Im Folgenden werden daher zunächst die Spezifika des deutschen Bildungssystems und des durch die BRK vorangetriebenen Wandels näher beleuchtet, sowie hieran anschließend die theoretischen Konzepte Bourdieus und Holzkamps und ihr praktischer Bezug zum Forschungsgegenstand exemplarisch an ausgewählten Forschungsarbeiten dargelegt.

\subsection{Die BRK und das deutsche Bildungssystem}

Die argumentative Grundlage der BRK bildet ein Paradigmenwechsel im Verständnis von Behinderung. Wurde Behinderung lange Zeit individuell in der betroffenen Person verortet und aus einer medizinischen Sicht verstanden, werden nun explizit die zu einer Behinderung führenden Barrieren berücksichtigt. Behinderung entsteht demzufolge aus der Wechselwirkung zwischen Beeinträchtigungen und einstellungs- und umweltbedingten Barrieren, die eine vollumfängliche Teilhabe verhindern (Art. 1 Unterabsatz 2 BRK). Die BRK fordert daher einen Wandel in der Betrachtung von und dem Umgang mit Behinderung. Mit dem Blick auf die praktische Umsetzung im Bildungsbereich, bedeutet dies nicht weniger als die Forderung nach einem grundlegenden Umbau des deutschen Bildungssystems (Kroworsch 2017, 6).

Dieser grundlegende Umbau wird vor allem deshalb notwendig, da dem deutschen Bildungssystem das Leistungsprinzip zugrunde liegt (Geissler 2014, 333). Es legitimiert den Erfolg oder Misserfolg nach der jeweils erbrachten Leistung in einem System, das formal an alle die gleichen Anforderungen stellt (Geissler 2014, 333f.; Bourdieu und Passeron 2007, 95f.). Dass mit dieser Annahme der vermeintlichen Gleichbehandlung aller, die eigentliche Ungleichheit erst entsteht, wird deutlich, wenn im Sinne der BRK die Rahmenbedingungen des Bildungserwerbs mitberücksichtigt werden. Diese Rahmenbedingungen stellen sich als Barrieren vielfältiger Art dar, die beeinträchtigte Menschen beim Bildungserwerb behindern. Mechanismen der Exklusion werden ohne die Berücksichtigung der Rahmenbedingungen verschleiert (Bourdieu und Passeron 2007, 98f.), der lebensweltliche Hintergrund der Betroffenen ausgeblendet (ebd., 24f.).

Das Bildungssystem erfüllt damit eine Platzierungsfunktion, also die Zuweisungeines gesellschaftlichen Status innerhalb der Gesellschaft (Geissler 2014, 333f.). Die Begründungslogik der Leistungsgerechtigkeit stellt eine wichtige Bedingung der gesellschaftlichen Anerkennung und innersystemischen Handlungsbegründung dar. Es wird einer Norm gefolgt, welche die Ungleichheit vermeintlich plausibel erklärt (Bourdieu und 
Passeron 2007, 95). Insofern ist durch den geforderten Paradigmenwechsel der BRK und der Dominanz des Leistungsprinzips im deutschen Bildungssystem (Sturm 2015, 27f.) von einem Wandel besonderer Intensität auszugehen. Dies zeigt sich nicht zuletzt in der kontroversen Debatte um das Thema Inklusion im Bildungssystem (Lindmeier und Lindmeier 2012, 179; Löser und Werning 2015). Die BRK fordert deshalb ausdrücklich die entsprechende Qualifizierung der Lehrkräfte (Art. 24 Abs. 4 BRK) und damit ein Erlernen von neuen Handlungspraktiken für eine Inklusive Erwachsenenbildung.

\subsection{Habitus und Feld}

Der Begriff des Habitus beschreibt den inkorporierten Bestandteil einer Person, der in Form von gesammelten Erfahrungen in einer sozialen Umgebung geprägt wurde und damit eine oft unbewusste Vorlage für Wahrnehmung, Bewertung und Handlung bereitstellt (Bourdieu 2005, 277ff.). Im Falle der Lehrkräfte stellt diese soziale Umgebung das Bildungssystem bzw. die Erwachsenenbildung dar. Durch die erfahrungsbedingte Abhängigkeit des Habitus und durch die allmähliche Verinnerlichung von Handlungsroutinen eröffnet sich aber auch die Möglichkeit, durch neue Erfahrungen, einmal verfestigte Handlungsroutinen aufzubrechen (Bourdieu 2004, 177ff., 206ff.). Der Habitus kann daher als ein Spielraum an Möglichkeiten und Grenzen der Handlungspraxis einer Person verstanden werden. Dies wiederum spiegelt sich unmittelbar in Erfahrungen, Wissen und Gütern wider, auf die eine Person zurückgreifen kann. Diese potenziell einsetzbaren Ressourcen bezeichnet Bourdieu als Kapital (Bourdieu 2006, 112f.).

Bourdieu trennt sein Konzept des Habitus nicht in unterschiedliche Sphären der sozialen Praxis auf, vielmehr ist der Habitus in seiner jeweiligen Ausformung als ganzheitlicher Bestandteil einer Person zu verstehen, die je nach sozialer Situation unterschiedliche Wirkung entfalten kann (Bourdieu 2005, 279ff.). Für die hier vorgenommene Untersuchung soll jedoch der professionelle Habitus im Zentrum der Betrachtung stehen. Diese Ergänzung des Professionellen zum Habitus steht für eine, dem Beruf entsprechende Handlungskompetenz (Arabin 1996, 11). Keinesfalls bedeutet dies, dass sich der Habitus trennscharf in einen beruflichen und einen nicht beruflichen Bestandteil aufspalten ließe. Mit der Betonung des Professionellen soll jedoch verdeutlicht werden, dass die Analyse der Handlungspraxis in einem jeweiligen beruflichen Kontext im Zentrum der Betrachtung steht.

Den Überlegungen Werner Helsper und Rudolf Tippelt $(2011,272)$ zum Thema Profession und Professionalität folgend, soll Professionalität über die Handlungsund Anforderungsstruktur und damit nicht nur vor dem Hintergrund einer einmal durchlaufenen beruflichen Ausbildung verstanden werden. Hierdurch wird es möglich, 
verschiedene pädagogische Berufe und unterschiedliche Beschäftigungsverhältnisse einzubeziehen (Helsper und Tippelt 2011, 272; Lindmeier und Weiß 2017, 3ff.). Für eine Untersuchung, die sich auf die Lehrkräfte im Berufsfeld der Erwachsenenbildung konzentriert, ist dies von besonderer Bedeutung, da die Gruppe der in diesem Bereich Lehrenden als sehr heterogen angesehen werden kann (Martin et al. 2016, 14ff.).

Trotz dieser heterogen strukturierten Gruppe der Lehrkräfte, bildet die Erwachsenenbildung ein eigenes Feld, in dem eine bestimmte Handlungslogik vorherrscht. Die Passung zwischen Habitus und Feld definiert dabei die Möglichkeiten des Handelns (Bourdieu 2005 279ff.). Diese Passung ergibt sich aus dem Besitz und dem adäquaten Umgang mit dem Kapital, dessen Ausdruck der Habitus ist (ebd., 281). Auf diese Weise wird die Position festgelegt, die eine Person in Relation zu anderen Personen im Feld einnehmen kann (ebd.). Die Feldstruktur wird durch die Personen festgelegt, welche die Bestimmungshoheit im Feld innehaben und der daraus entstehenden Struktur, welcher diese gleichermaßen unterworfen sind (Bourdieu 2004, 210ff.). Ein tiefgreifender Wandel dieser Strukturen kann dazu führen, dass eine Anpassung des Habitus hieran misslingt (ebd., 207f.). Die einmal eingeübten Handlungsroutinen des Habitus können auf diese Weise dysfunktional werden (ebd.).

Für die Untersuchung der Handlungspraxis bedeutet dies, dass eine Reflexion vorhandener professioneller Kompetenzen und Bedarfe der Lehrkräfte und der Rahmenbedingungen nötig ist. Klaus Meisel (2012) weist darauf hin, dass den in der Erwachsenenbildung tätigen Pädagog*innen der Mangel an inklusiven Angeboten nicht vorgeworfen werden könne. Verantwortlich seien vielmehr die strukturellen Bedingungen, die der systematischen Professionalisierung der Lehrkräfte entgegenstehen (Meisel 2012, 20f.). Diese beschränkenden Faktoren nennt auch Christian Papadopoulos (2012) in seiner Darlegung der didaktischen Herausforderungen einer barrierefreien Gestaltung von Bildungsangeboten. Jedoch betont er, dass es für die Erwachsenenbildung nicht der grundsätzlichen Neuerfindung einer inklusiven Didaktik bedarf, sondern der Rückgriff auf vielversprechende Ansätze der Erwachsenenbildung möglich ist (Papadopoulos 2012, 38).

Eine mögliche Modifikation des Habitus untersucht auch Wiltrud Gieseke (1989), wenn auch vor einem anderen Hintergrund. Von ihr werden die Mechanismen des beruflichen Einarbeitens beim Übergang der Lehrkräfte in die Praxis der Erwachsenenbildung untersucht (Gieseke 1989, 40f.). Hierbei kann von einem ähnlichen Prozess im Falle von Wandlungsprozessen ausgegangen werden, der unmittelbar Folgen für das berufliche Feld der Erwachsenenbildung hat und ebenfalls einen Lernprozess von den Lehrkräften verlangt. Gieseke $(1989,96)$ geht von einem praxisgebundenen Habitus der Lehrkräfte aus. Im Mittelpunkt steht aber nicht eine umfassende Typisierung eines individuellen oder allgemeinen Habitus der Lehrkräfte, sondern die Beschreibung des Modus der Aneignung (Gieseke 1989, 151). Ein Ergebnis ist, dass der bestehende Ha- 
bitus der Lehrkräfte von den beruflichen Anforderungen überformt wird, indem die bestehende beruflichen Struktur auf den Habitus der Lehrkräfte einwirkt (ebd., 263f.). Individuelle Eigenheiten bleiben trotz dieser Überformung bestehen und zeigen sich in den Mustern der Aneignungsmodi der Lehrkräfte, also in der Art und Weise, wie diese ihr neues Umfeld erschließen (ebd., 264f.).

Die skizzierte theoretische Perspektive Bourdieus ermöglicht es, eine Analyse der Mechanismen des professionellen Habitus innerhalb von Wandlungsprozessen vorzunehmen. Das Zusammenspiel von Habitus und Feld dient dabei als Erklärung für das Verhalten in neuen sozialen Situationen, das sich an früheren Handlungsroutinen orientiert und mitunter eine Anpassung verhindert oder aber auch erleichtert. Besonders die dargelegte Arbeit Giesekes (1989), lässt auch einen Anschluss an das theoretische Konzept Holzkamps (1995) zu, scheint der Habitus doch mit einer bestimmten Art von Lernprozess verbunden zu sein.

\subsection{Subjektwissenschaftliche Lerntheorie}

Nach der Perspektive der subjektwissenschaftlichen Lerntheorie Klaus Holzkamps (1995), wird Lernen als eine Handlungspraktik verstanden, um eine bestehende Handlungsproblematik zu bewältigen (Holzkamp 2004, 29). Das Subjekt erweitert seine Handlungsfähigkeiten, indem es die vorliegende Handlungsproblematik in einer Lernschleife auflöst. Das Lernen erfolgt nicht aufgrund der Initiierung von dritter Seite. Entscheidend ist die Lösung des Handlungsproblems durch die Lernenden selbst (Holzkamp 2004, 29f.).

Je nach Art des Handlungsproblems kann der Lernprozess höchst unterschiedlich ablaufen. Tritt während einer Handlung ein Problem auf, welches im Handlungsverlauf unmittelbar gelöst werden kann, kommt es zum Mitlernen (Holzkamp 1995, 183). Dieses Lernen erfolgt meist unbewusst. Erst wenn der Handlungsverlauf zur Lösung der Problemkonstellation durch den Akteur unterbrochen werden muss, setzt eine bewusste Lernschleife ein, die den Lernprozess zwischen dem Erkennen und der Lösung des Handlungsproblems beschreibt (ebd., 183). Erscheint ein Problem vor dem Hintergrund bisherigen eigenen Wissens als unüberwindbar, entsteht ein begründetes Lerninteresse. Diese Erfahrung der Unzulänglichkeit, bedingt durch das nicht ausreichende eigene Wissen und dem zur Problemlösung nötigen Wissen, ist entscheidend für eine entsprechende Lernmotivation (ebd., 214f.).

Erfolgt das Lernen defensiv unter der Prämisse der Abwehr von negativen Konsequenzen, die sich aus der Handlungsproblematik ergeben könnten, so kann es sein, dass das Lernen vorgetäuscht oder lediglich unter dem geringsten Aufwand und entsprechend großem inneren Widerstand vollzogen wird (ebd., 192f.). Es werden Abwehrre- 
aktionen hervorgerufen, die einer tatsächlichen Überwindung des Handlungsproblems im Wege stehen. Wird dagegen deutlich, dass mit einem dem Lernenden subjektiv angemessenen Lernaufwand eine Überwindung des Handlungsproblems möglich wird, verbindet der Lernende hiermit eine für ihn nutzbringende Verfügungserweiterung der Handlungsmöglichkeiten und entwickelt eine entsprechende Lernmotivation (ebd., 190f.). Dies wird als expansives Lernen bezeichnet. Mit diesem Verständnis von Lernen bietet die subjektwissenschaftliche Lerntheorie die Möglichkeit, die konkreten Handlungsproblematiken, hier die Gestaltung inklusiver Bildungsangebote durch die Lehrkräfte, für eine Analyse zugänglich und erklärbar zu machen.

In ähnlicher Weise werden in der Untersuchung von Claudia Schepers (2014) die Lernwiderstände von Lehrkräften im Zuge von Fortbildungen betrachtet (Schepers 2014, 9f.), die als Orte der Professionalitätsentwicklung aufgefasst werden (ebd., 11). Ergebnis dieser Untersuchung ist, dass bei Lernhandlungen die gegebenen Rahmenbedingungen mitbedacht werden müssen. Lernen sei nach Holzkamp in bestimmte Macht- und Herrschaftsstrukturen eingebettet aus denen Lernwiderstände resultieren können. Ihre Auswertung bestätigte diese Annahme. Sehr wohl können solche Strukturen Lernwiderstände hervorrufen, müssen dies aber nicht zwingend (Schepers 2014, 194). An dieser Stelle wiederum, ließe sich mit Bourdieu ansetzen, indem man die zugehörigen Feldkontexte einer genaueren Analyse unterziehen könnte.

\subsection{Synergieeffekte der Theorien von Bourdieu und Holzkamp}

In der Anwendung beider theoretischer Perspektiven liegt die Möglichkeit, den Umgang der Lehrkräfte mit einer Handlungsproblematik in Form des Erlernens neuer Handlungsmöglichkeiten als spezifische Form des sozialen Handelns im Feldkontext der Erwachsenenbildung, vor dem Hintergrund ihres professionellen Habitus, zu betrachten. Auf diese Weise lassen sich die beiden theoretischen Ansätze, trotz ihrer unterschiedlichen Ausrichtungen, miteinander in Beziehung setzen und bieten damit die Möglichkeit, Leerstellen bei der empirischen Analyse zu füllen (Bremer und Trumann 2013, 334f.). Der quasi Determinismus des Habitus Bourdieus, wird durch Holzkamps potenziell mögliche Verfügungserweiterung, in Form eines expansiven Lernprozesses aufgelockert bzw. die Gründe hierfür in der Art verortet, wie entsprechende Lernprozesse gestaltet sind. Ebenso liefert aber auch Bourdieu eine Erklärung dafür, warum selbst bei optimalen Bedingungen im Sinne Holzkamps, aufgrund sozialstruktureller Phänomene eine Verfügungserweiterung nicht gelingt.

Hierbei muss jedoch beachtet werden, dass den beiden theoretischen Ansätzen ein anderes Erkenntnisinteresse zugrunde liegt (ebd., 335). Während Bourdieus Ansatz darauf ausgerichtet ist, zu erklären wie soziale Strukturen reproduziert werden und wo- 
durch die Handlungsmöglichkeiten der jeweiligen Akteure eine Begrenzung erfahren, geht es Holzkamp vielmehr um den aktiven und bewussten Prozess der Erweiterung von Handlungsmöglichkeiten (ebd., 355). Letztendlich resultiert dieser Gegensatz aus unterschiedlichen wissenschaftstheoretischen Positionen Bourdieus und Holzkamps, die nicht unbeachtet bleiben dürften (ebd.). Eine kombinierte Anwendung als Analyseinstrument im Rahmen des hier umrissenen Sensitizing Concept scheint jedoch sinnvoll, nicht nur, um die etwaigen Leerstellen in den theoretischen Ausarbeitungen aufzufangen, sondern auch, um die Reflexionsprozesse über und entlang des Sensitizing Concept durch die unterschiedlichen Perspektiven zu unterstützen und hierdurch stets auch alternative Erklärungsmuster zu berücksichtigen.

\section{$4 \quad$ Erhebungsmethode und Durchführung}

Es wurden bereits drei Gruppendiskussionen mit Lehrkräften und Personal der Leitungsebene durchgeführt. Hieran sollen im Herbst 2019 vertiefende Einzelinterviews anschließen. Das Ziel der vorgeschalteten Gruppendiskussionen war es, das Feld möglichst breit zu erfassen und einen ersten Überblick zu gewinnen. Daher erfolgte auch die Einbeziehung der Leitungsebene, um die Rahmenbedingungen detaillierter abbilden zu können. Durch die unterschiedlichen Erhebungsmethoden schließlich, sollen die Stärken beider Verfahren zur Geltung gebracht werden (Loos und Schäffer 2001, 41).

Durch die Gruppendiskussionen sollen die Gemeinsamkeiten und Unterschiede der berichteten Handlungspraxis und deren subjektive Begründungen für die Untersuchung zugänglich gemacht werden (Schepers 2014, 210f.). Des Weiteren ermöglichen die Gruppendiskussionen auch, die jeweiligen Positionierungen im Feld der Erwachsenenbildung in den Diskussionen nachzuvollziehen. Innerhalb der Diskussion kann vor diesem Hintergrund auch nachverfolgt werden, welches Kapital von den Teilnehmenden im Zusammenhang mit dem sich vollziehenden Wandel als nützlich oder überholt angesehen wird. Im Rahmen des Theoretical Sampling der Grounded Theory sollen sich den Gruppendiskussionen Einzelinterviews anschließen, um die vorgefundenen Phänomene vertiefend untersuchen zu können. Im Forschungsprozess wechseln sich dabei Datenerhebung und Auswertung ab, bis dass interessierende Phänomen analytisch beschreibbar geworden ist (Charmaz 2014, 192ff.). Auftauchende neue Fragen, die sich aus der Analyse ergeben und anhand der bestehenden Daten nicht beantwortet werden können, führen zu einer erneuten Erhebung von Daten und deren Analyse.

Die folgende Beschreibung der Spezifika der Erhebung wird sich auf die problemzentrierten Gruppendiskussionen (Kühn und Koschel 2018) beschränken, da zum Erscheinen dieses Artikels noch keine Einzelinterviews stattgefunden haben und die 
Darstellung erster relevanter Befunde sich ausschließlich auf die Gruppendiskussionen bezieht. Die Methode der problemzentrierten Gruppendiskussion wurde von Thomas Kühn und Kay-Volker Koschel (2018) in Anlehnung an das problemzentrierte Interview (Witzel 2000) entwickelt. Zentrales Element ist der Leitfaden, der die Problemzentrierung am Thema entlang, bei einer ansonsten offen angelegten Gruppendiskussion, gewährleisten soll (Kühn und Koschel 2018,95). Durch gezieltes Nachfragen aus unterschiedlicher Perspektive und unter dem Rückgriff auf die Aussagen der Teilnehmenden wird versucht, in eine vertiefende Bearbeitung der interessierenden Thematik seitens der Teilnehmenden zu gelangen (ebd., 96f.). Interessierende thematische Bezüge können dabei mit gruppendynamischen Prozessen ins Verhältnis gesetzt werden (ebd., 274).

Insgesamt wurden drei Gruppendiskussionen mit einer Länge von je 90 Minuten geführt. Theoretical Sampling kam in diesem ersten Schritt nicht zur Anwendung. Bei den Gruppendiskussionen handelte es sich um eine gezielte Auswahl von Lehrkräften und leitendem Personal aus Alphabetisierung und Zweitem Bildungsweg aus unterschiedlichen Bundesländern, die bereits Erfahrungen mit dem Unterricht von behinderten und nichtbehinderten Teilnehmenden hatten.

An der ersten Gruppendiskussion (A) nahmen sechs Teilnehmende aus drei verschiedenen Einrichtungen teil. Eine Person aus dem Leitungsbereich, zwei Lehrkräfte aus einer weiteren Einrichtung und drei Lehrkräfte wiederum aus einer anderen Einrichtung. Die zweite Gruppendiskussion (B) setzte sich aus fünfTeilnehmenden zusammen. Hierbei stammte lediglich eine Lehrkraft aus einer anderen Einrichtung. Die weiteren drei Lehrkräfte sowie die Leitungskraft kamen aus derselben Einrichtung. Die dritte Gruppendiskussion (C) stellt einen Sonderfall dar. Anders als geplant, nahmen an dieser Diskussion 18 Personen aus verschiedenen Einrichtungen teil. Bei den Teilnehmenden handelte es sich lediglich bei einer Person um eine Lehrkraft, die übrigen Teilnehmenden kamen aus dem Bereich der Leitung. Die Teilnehmendenzahl überstieg deutlich die als ideal angesehene Menge in diesem Befragungsformat (ebd., 76f.). Die Gruppendiskussion lief jedoch sehr strukturiert ab und lieferte ein reichhaltiges Datenmaterial, wobei sich eine diskutierende Kerngruppe herausbildete und die übrigen Teilnehmenden die Diskussion ohne Beiträge verfolgten. Unter der Berücksichtigung der besonderen Erhebungssituation wurde diese Gruppendiskussion in die Auswertung einbezogen.

Vor der eigentlichen Initialfrage an die jeweilige Gruppe wurde das Verständnis von inklusiver Bildung als gemeinsames und gleichberechtigtes Lernen von behinderten und nichtbehinderten Lernenden präzisiert. Es folgte die Aufforderung an die Teilnehmenden, über die Erfahrungen aus ihrer Lehrtätigkeit zu diskutieren. Nachdem ein erster Austausch stattgefunden hatte, wurde nach einer halben Stunde ein neuerlicher Impuls gesetzt, indem gefragt wurde, mit welchen Methoden die Teilnehmenden 
den Unterricht mit behinderten und nichtbehinderten Teilnehmenden in ihren Kursen konkret umsetzten. Nach einer weiteren halben Stunde wurde als ein weiterer Impuls ein vier Minuten langes Video der VHS-Mainz gezeigt, in dem es um die Umsetzung von Inklusion vor dem Hintergrund der BRK ging. Im Anschluss hieran wurde gefragt, ob die Teilnehmenden hierzu generell etwas sagen möchten oder Anknüpfungspunkte zu ihrer eigenen Arbeit finden.

\section{Erste relevante Befunde aus den Gruppendiskussionen}

Die Grundlage der Auswertung bilden die Transkriptionen der Audioaufnahmen der Gruppendiskussionen. Aufgrund der großen Datenmenge und der Komplexität durch mehrere Diskutanten, wurde auf die Software F4 für die Transkription und MAXQDA für die Auswertung zurückgegriffen (Kühn und Koschel 2018, 188). Die einzelnen Wortbeiträge wurden hierbei personalisiert erfasst, um eine Nachvollziehbarkeit zu gewährleisten (ebd., 200). Eine erste Durchsicht des Materials ist bereits erfolgt, wobei mit dem offenen Codieren nach dem Vorgehen der Grounded Theory begonnen wurde. Insofern kann die hier vorgenommene Präsentation als ein Einblick in den laufenden Forschungsprozess verstanden werden. Im Folgenden werden erste relevante Befunde aus dem Auswertungsprozess präsentiert, wobei zunächst einzelne thematisch zusammenhängende Passagen codiert wurden (Charmaz 2014, 109; Corbin und Strauss 2015, 86f.). Im Anschluss an das zuvor skizzierte Sensitizing Concept wurden hierfür prägnante Passagen aus den Diskussionen ausgewählt.

\subsection{Handlungsproblematiken und Lernhandlungen}

In der Diskussion unmittelbar vor der folgenden Passage ging es um die allgemeine Unsicherheit der Diskutierenden im Falle eines Brandes. Folgende Passage ist der Beitrag einer Lehrkraft des Zweiten Bildungsweges.

Lehrkraft (A1): »[W]o wir den Rollstuhlfahrer runtertragen mussten, weil der Fahrstuhl kaputt war. Gott sei Dank war es so, dass eine Schülerin da war, die mal eine Ausbildung zur Krankenschwester gemacht hatte. Ich hätte nicht gewusst, wo ich den jetzt anpacken kann, er hätte es mir vielleicht selbst sagen können ne, aber so denk ich manchmal. Ich habe auch einen Gehbehinderten in meinem Kurs selbst, der mal gestürzt ist, [...] ich weiß nicht ob's so ne, ja Erste-Hilfe oder, also so praktische Basics gibt vielleicht einfach, ne? Ist ja natürlich auch sehr situations- und einschränkungsabhängig $[\ldots] \cdot \ll$ 
Es bleibt bei dieser Passage im Unklaren, ob die letztgenannte mögliche Handlungsstrategie erst im Anschluss an die betreffende Situation entstanden ist oder schon vorhanden war. Ungeachtet dessen wünscht sich die Lehrkraft allgemeine Handlungsanweisungen für den korrekten Umgang mit behinderten Kursteilnehmenden, um daraufhin in einem Nachsatz diesen Wunsch analog zu dem ersten Abschnitt der Passage zu entkräften, mit dem Hinweis auf die Situationsabhängigkeit und Form der Behinderung. Obwohl also eine erfolgversprechende Handlungsstrategie genannt wird, welche in Bezug auf das Thema Behinderung eine individualisierte Lösung zulassen würde, nämlich den behinderten Kursteilnehmenden selbst zu fragen, wird ein allgemeiner Lösungsansatz gefordert, welcher der Lehrkraft selbst aber wenig erfolgversprechend erscheint.

Die sich bereits zu Beginn der Diskussionen zeigende Unsicherheit mit dem Thema Behinderung, setzt sich auch in dieser Passage fort. Erstaunlich ist hierbei, dass die Teilnehmenden auch dann von dieser Unsicherheit betroffen waren, wenn sie über erfolgversprechende Handlungsstrategien verfügen. Eine mögliche Erklärung ist, dass die Anwesenheit eines behinderten Kursteilnehmenden die vorherrschende Handlungslogik des Feldes derartig stört, dass neue Handlungsstrategien in einer Abwehrhaltung über den Prozess des defensiven Lernens in den professionellen Habitus aufgenommen werden und eine tatsächliche Erweiterung der Handlungsmöglichkeiten auf diese Weise blockiert wird. Die erste ausgewählte Passage steht dabei exemplarisch für dieses widersprüchliche, aber dennoch subjektiv erklärbare Verhalten.

Als Kontrast hierzu wird die behinderte Person in der nächsten Passage, nach anfänglichem Widerstand, als Expert*in ihrer Beeinträchtigung akzeptiert. Auch entgegen des eigentlich für sie geplanten Vorgehens der für sie zuständigen Betreuung, wird ihr zugestanden, nach ihren eigenen Vorstellungen zu lernen und am Kurs teilzunehmen. In dieser Passage berichten eine Person aus dem Leitungsbereich und eine Lehrkraft aus dem Bereich des Zweiten Bildungsweges über einen erblindeten Teilnehmenden im Kurs.

Leitung (B1): »Ja, das war nämlich schon ziemlich schwierig mit Person X. [...] insofern, weil wir also wir sind darauf nicht vorbereitet letztendlich, ne. Also wir sind weder geschult noch ausgebildet noch in irgendeiner Weise auf solche Sachen vorbereitet $[\ldots] . \ll$

Lehrkraft (B2): »[D]ie Person X hat alles auditiv aufgegriffen und alles Optische stand ihm nicht zur Verfügung und trotzdem hat er den Abschluss gemacht. [...] ich weiß noch, [...] dass die Betreuerin immer ganz erstaunt war, die einmal die Woche oder einmal im Monat kam, ich weiß es gar nicht mehr: >Das ist ja komisch, also er macht alles so mit und ja aber er muss doch schreiben können oder wir wollen gerne, dass er Brailleschrift lernt< und so weiter und er drückt sich. Ja, wenn er's nicht braucht offensichtlich braucht er' s nicht. [...].« 
Aus der hierauf folgenden längeren Erzählpassage der Lehrkraft wird deutlich, dass der Teilnehmende als Hilfsmittel lediglich die Sprachausgabe und Sprachsteuerung seines Smartphones nutzt. Im weiteren Verlauf der Gruppendiskussion zeigte sich dann, dass die Lehrkraft das Smartphone auch über diesen konkreten Fall hinaus als Hilfsmittel im Kurs einsetzt.

Lehrkraft (B2): $\gg$ Also wie Voice Over sehr gut ging mit Person X, [...] so in der Art machen wir das auch mit Person Y, [...] der nicht freisprechen kann, er schickt mir einfach Sprachmessages (Zwischenfrage: Im Unterricht?) nee nach dem Unterricht [...] er musste auch mal einen kleinen Vortrag halten, den hat er ganz und gar in 45 einzelnen Sprachmessages geschickt $[\ldots] . \ll$

Die hier kontrastierten Passagen weisen einen sehr unterschiedlichen Verlauf in der Lösung von Handlungsproblematiken auf. Der professionelle Habitus wird in beiden Fällen herausgefordert, da eine Situation vorherrscht, für die keine Handlungsroutinen vorliegen. Im ersten Fall wird die Verantwortung für den weiteren Handlungsverlauf abgegeben. Das Handlungsproblem wird hierbei zwar bewusst, die Handlungsproblematik wird jedoch nicht im Sinne einer Verfügungserweiterung der eigenen Handlungsmöglichkeiten gelöst. Im zweiten Fall wird die aus der anfänglich bestehenden Handlungsproblematik hervorgegangenen Erweiterung der Handlungsmöglichkeiten deutlich, da das einmal erlernte Wissen um den Gebrauch des Smartphones als Lernhilfe auch in einem anderen Kontext wieder angewendet wird. Unklar bleibt hierbei aber, ob es sich um ein Mitlernen oder tatsächlich um expansives Lernen handelt.

\subsection{Irritationen des professionellen Habitus?!}

In der folgenden Passage berichtet eine Lehrkraft aus der Alphabetisierung von einem Teilnehmenden, der noch im jugendlichen Alter ist und eigentlich eine Sonderschule besucht. Im weiteren Gesprächsverlauf stellt sich heraus, dass auf Drängen der Eltern ein zusätzlicher Besuch eines Alphabetisierungskurses in der Erwachsenenbildung ermöglicht wurde.

Lehrkraft (A2): »[A]us irgendwelchen Gründen, ich weiß selbst nicht aus welchen ist der in diesem Kurs gelandet [...] und als er zu uns gekommen ist, vor etwas über einem Jahr, konnte er kein Wort lesen. [...] er konnte gerade die Buchstaben, er wusste, wie sein Name anfängt, das wars aber auch. Und es ist mittlerweile tatsächlich so, dass er Worte lesen kann [...] find ich schon wirklich erstaunlich, also denn, also wir sind zu zweit klar, aber wir sind keine Sonderpädagoginnen und das ist auch nicht mein 
Verdienst, sondern der meiner Kollegin. Ich habe am Anfang sogar gesagt, der gehört hier gar nicht her, vom Alter nicht und was will der hier eigentlich und außerdem passt das gar nicht $[\ldots] . \ll$

Die obige Passage zeigt, wie der professionelle Habitus der Lehrkraft durch einen behinderten Teilnehmenden irritiert wurde. Anfänglich reagiert die Lehrkraft hierauf ablehnend und ist deshalb umso erstaunter darüber, dass bei dem Kursteilnehmenden ein Lernerfolg erzielt wird. Hierbei zeigt sich, dass der jugendliche Kursteilnehmende eigentlich nicht in den Feldkontext der Erwachsenenbildung passt und deshalb zunächst abgelehnt wird. Im Anschluss an die Ausführung der Lehrkraft wurde seitens der Moderation nachgefragt, wie sich die Lehrkraft, diesen Erfolg erklären kann.

Lehrkraft (A2): »[D]ass es daran liegt, dass er eben wie ein Erwachsener behandelt wird, mit Respekt auch ne und er hat bei uns nicht irgendwie nen Stempel >das kannst du sowieso nicht< oder so, sondern äh durch Beharrlichkeit und wirklich dann ihn immer wieder damit, mit Lesen konfrontieren, ja dann in den sechs Stunden die Woche auf jeden Fall, das hat offensichtlich Wirkung gezeigt, denk ich [...].«

Der Erfolg bezieht sich nicht nur auf den individuellen Lernerfolg des Teilnehmenden, sondern auch auf das erwachsenenpädagogische Vorgehen. Es scheinen hier die Rahmenbedingungen des Lernens zu sein, die den Ausschlag geben. Die Überwindung der Handlungsproblematik erfolgt hier im Sinne einer Bewusstwerdung der Stärken der eigenen Professionalität vor dem Hintergrund neuer Anforderungen. Hierzu passt folgende Aussage einer Leitungskraft, die die Stärken der Erwachsenenbildung betont.

Leitung (C1): »[D]a habe ich aber den Eindruck, dass ähm viele Unterrichtende dann auch sehr bemüht sind und eben grundsätzlich sehr wertschätzenden Umgang haben mit allen Teilnehmenden und ich glaube, das ist die Stärke des Zweiten Bildungswegs. Ähm, dass wir gar nicht so genau hingucken, was ist da eigentlich jetzt das größte Problem vorneweg, sondern wir gucken einfach den Menschen an und gucken, wie können wir zum Ziel kommen. Und das finde ich ist nen wesentlicher Bestandteil unserer Arbeit und ähm das finde ich nach wie vor sehr wichtig.«

Die Erwachsenenbildung und damit die Lehrkräfte verfügen somit über Stärken, die sich für die Inklusion nutzbar machen lassen. An dieser Stelle kann an die im Forschungsstand skizzierte Prämisse angeknüpft werden, dass es für die Erwachsenenbildung nicht einer grundsätzlichen Neuerfindung einer inklusiven Didaktik bedarf, sondern diese durchaus auf eigene Ansätze zurückgreifen kann (Papadopoulos 2012). Diese Fähigkeiten sind entsprechend Bourdieus (2005) theoretischen Konzepts Kapital, dessen 
richtige Verwendung in einem sich wandelnden Feld vielfach erst noch erlernt werden muss, womit sich wiederum ein Anknüpfungspunkt an die subjetwissenschaftliche Lerntheorie Holzkamps (1995) finden lässt.

\section{Fazit/Ausblick}

Zunächst bleibt festzuhalten, dass der hier vorliegende Beitrag lediglich einen Einblick in den noch nicht abgeschlossenen Forschungsprozess geben kann. Die präsentierten Schlussfolgerungen müssen daher in aller Vorsicht betrachtet werden. Dennoch lässt sich bereits jetzt ein wichtiger Befund festhalten. Die Vermutung, dass bereits Kurse in der Alphabetisierung und dem Zweiten Bildungsweg existieren, in denen behinderte und nichtbehinderte Kursteilnehmende gemeinsam und gleichberechtigt lernen, hat sich bestätigt. Auch wenn hier nur ein kleiner Ausschnitt aus Alphabetisierung und Zweitem Bildungsweg einer Untersuchung unterzogen wurde, wird deutlich, dass das Thema von Inklusion im Kontext von Behinderung längst in der Realität der Erwachsenenbildung angekommen ist und dies nicht nur in Kursen, die auf Inklusion angelegt oder an entsprechende Projekte zur Inklusion angedockt sind. Hierbei muss auf einen weiteren limitierenden Faktor der vorgestellten Arbeit hingewiesen werden. Es handelt sich um ein rein qualitatives Forschungsprojekt und darf daher nicht als statistisch repräsentativ angesehen werden. Der Sachverhalt an sich ist damit jedoch nicht infrage gestellt, sondern eröffnet vielmehr eine begründete Möglichkeit für eine größer angelegte Studie.

Nicht unerwähnt bleiben darf auch die starke normative Konnotation der BRK, welche den Ausgangspunkt der Untersuchung bildet. Zusammen mit der starken theoretischen Fundierung durch das beschriebene Sensitizing Concept, besteht dabei immer die Gefahr, den explorativen Forschungscharakter der Grounded Theory zu konterkarieren. Durch den transparenten Umgang mit dem Vorwissen und dem Bewusstsein um die Notwendigkeit eines Reflexionsprozesses über die vorgenommenen Interpretationen des Datenmaterials, soll dieser Gefahr aber entgegengewirkt werden. Auf diese Weise besteht für den Forschenden selbst und Außenstehende die Möglichkeit eines kritischen Umgangs mit dem Forschungsprozess. Darin liegt schließlich die Stärke einer konstruktivistisch verstandenen Anwendung der Grounded Theory, was gleichzeitig aber auch bedeutet, zu akzeptieren, dass der Forschungsprozess als solcher nie durch das Vorwissen des Forschenden unbeeinflusst bleibt.

Abseits von den theoretischen, normativen und rechtlichen Vorannahmen zeigen die Daten der Gruppendiskussionen jedoch deutlich den hohen Bedarf der befragten Lehrkräfte nach zusätzlicher Qualifizierung für inklusive Bildungsangebote. Für die Lehrkräfte selbst steht hierbei in erster Linie nicht unbedingt die Umsetzung der 
BRK im Vordergrund, sondern vielmehr die konkreten Handlungsprobleme, denen sie innerhalb ihrer beruflichen Tätigkeit begegnen. Hieraus ergibt sich wiederum eine Bestätigung der bildungspolitischen Forderung durch die BRK, das Lehrpersonal entsprechend für inklusive Bildungsangebote fortzubilden. Dies gilt im Besonderen auch für den eher vernachlässigten Bildungssektor der Erwachsenenbildung.

Als vorläufiges Fazit der vorgenommenen Betrachtung der Daten aus den Gruppendiskussionen lässt sich anschließen, dass die Lehrkräfte und die Leitungsebene damit befasst sind, behinderte Kursteilnehmende in ihrer Lehre gleichberechtigt zu berücksichtigen. Unter dem Rückgriff auf das Sensitizing Concept lässt sich folgern, dass der professionelle Habitus der Lehrkräfte unter Anpassungsdruck gerät, was sich in gehäuftem Auftreten von Handlungsproblematiken im Praxisalltag äußert. Hierbei besteht immer die Gefahr, dass der bestehende professionelle Habitus im Zuge der neuen Anforderungen eine dysfunktionale Wirkung entfaltet. In dieser Situation wäre es daher äußerst kontraproduktiv, den Lehrkräften Fortbildungen anzubieten, die sich nicht in genügender Weise an deren tatsächlichen Handlungsproblematiken orientieren und praxisfremde Handlungsstrategien propagieren. Eine nicht unwahrscheinliche Folge hieraus wäre eine defensiv begründete Lernhandlung, welche eine Erweiterung der Handlungsverfügung blockieren könnte.

Es gilt vielmehr anzuerkennen, dass bereits vielfältige Erfahrungen und erfolgversprechende Handlungsstrategien von Lehrkräften im Umgang von behinderten und nichtbehinderten Lernenden in der Erwachsenenbildung bestehen. Es besteht daher die Möglichkeit, gelingende Handlungspraktiken inklusiver Erwachsenenbildung direkt aus der Praxis zu gewinnen und im Rahmen von Fortbildungen für die Breite der Lehrkräfte in der Erwachsenenbildung zugänglich zu machen. Hervorzuheben ist hierbei, dass gemeinhin aus der Praxis gewonnene Erkenntnisse eine große Akzeptanz bei den Lehrenden besitzen und daher von besonderer Bedeutung für Qualifizierungsmaßnahmen sind.

Dabei bietet gerade die Erwachsenenbildung mit ihren reichhaltigen eigenen pädagogischen Konzepten, die durch eine größere Offenheit gekennzeichnet sind, die Möglichkeit, die in der Praxis angewendeten Handlungsstrategien für eine Inklusive Erwachsenenbildung an die theoretischen Prämissen der allgemeinen Erwachsenenbildung rückzubinden. Es kann auf bereits bekannte Konzepte verwiesen und so der vermutete Anpassungsdruck des professionellen Habitus der Lehrkräfte reduziert werden. Es darf vermutet werden, dass sich dies positiv auf Akzeptanz und gelingende Umsetzung inklusiver Bildungsangebote auswirken würde.

Wie sich in den ersten relevanten Befunden zu der hier präsentierten Untersuchung zeigt, besteht auf diese Weise die Chance, Inklusion durch die Anwendung der von den Lehrkräften oftmals sogar schon beherrschten Techniken, zu fördern. Wie sich der hier dargelegte interpretative Gedankengang im weiteren Auswertungsprozess und im 
Rahmen des Theoretical Sampling weiterentwickelt, muss sich jedoch erst noch zeigen. Entscheidend hierfür sind die für den Herbst 2019 beabsichtigten Einzelinterviews, die stärker die individuelle Perspektive der Lehrkräfte und des leitenden Personals erfassen sollen.

\section{Literatur}

Ackermann, Karl-Ernst. 2017. »PPädagogische Professionalität` im Handlungsfeld inklusiver Erwachsenenbildung. Eine Problemskizze in vier Thesen."In Pädagogische Professionalität im Spannungsfeld von sonderpädagogischer Förderung und inklusiver Bildung, herausgegeben von Christian Lindmeier und Hans Weiß, 134-153. Weinheim: Beltz Juventa.

Arabin, Lothar. 1996. Unterrichtende an hessischen Volkshochschulen. Historische und empirische Analyse zur Arbeitssituation, zur Motivation und zu Fortbildungsproblemen. Frankfurt/M.: HVV.

Babilon, Rebecca. 2018. Inklusive Erwachsenenbildung mit Menschen mit Lernschwierigkeiten - eine qualitative Studie in England. Dissertation. Landau: Universität Koblenz-Landau, Campus Landau, Universitätsbibliothek.

Behindertenrechtskonvention (BRK). 2008. Gesetz zu dem Übereinkommen der Vereinten Nationen vom 13. Dezember 2006 über die Rechte von Menschen mit Behinderungen sowie zu dem Fakultativprotokoll vom 13. Dezember 2006 zum Übereinkommen der Vereinten Nationen über die Rechte von Menschen mit Behinderungen. In Bundesgesetzblatt Jahrgang 2008 Teil II Nr. 35, ausgegeben zu Bonn am 31. Dezember 2008.

BMAS - Bundesministerium für Arbeit und Soziales (Hrsg.). 2016. Zweiter Teilhabebericht der Bundesregierung über die Lebenslagen von Menschen mit Beeinträchtigungen. Teilhabe - Beeinträchtigung - Behinderung. Bonn: Referat Information, Monitoring, Bürgerservice, Bibliothek.

Bourdieu, Pierre. 2004. Meditationen. Zur Kritik der scholastischen Vernunft. Frankfurt/M.: Suhrkamp.

Bourdieu, Pierre 2005. Die feinen Unterschiede. Kritik der gesellschaftlichen Urteilskraft. 16. Auflage. Frankfurt/M.: Suhrkamp.

Bourdieu, Pierre 2006. Wie die Kultur zum Bauern kommt. Über Bildung, Schule und Politik. Hamburg: VSA-Verlag.

Bourdieu, Pierre, und Jean-Claude Passeron. 2007. Die Erben. Studenten, Bildung und Kultur. Konstanz: UVK-Verl.-Ges.

Bremer, Helmut, und Jana Trumann. 2013. »Politisches Lernen zwischen Holzkamp und Bourdieu.« In Politische Bildung zwischen Politisierung, Partizipation und politischem Lernen. Beiträge für eine soziologische Perspektive, herausgegeben von Helmut Bremer, Mark Kleemann-Göhring, Christel Teiwes-Kügler und Jana Trumann, 315-338. Weinheim: Beltz Juventa.

Charmaz, Kathy. 2014. Constructing Grounded Theory. 2. Auflage. Los Angeles: SAGE.

Corbin, Juliet M., und Anselm L. Strauss. 2015. Basics of Qualitative Research. Techniques and Procedures for developing Grounded Theory. 4. Auflage. Los Angeles: SAGE.

Geißler, Rainer. 2014. Die Sozialstruktur Deutschlands. 7. Auflage. Wiesbaden: Springer VS.

Gieseke, Wiltrud. 1989. Habitus von Erwachsenenbildnern. Eine qualitative Studie zur beruflichen Sozialisation. Oldenburg: Bibliotheks- und Informationssystem der Univ. Oldenburg.

Helsper, Werner, und Rudolf Tippelt. 2011. »Ende der Profession und Professionalisierung ohne Ende? Zwischenbilanz einer unabgeschlossenen Diskussion. «In Pädagogische Professionalität (Zeitschrift für Pädagogik, Beiheft 57), herausgegeben von Werner Helsper und Rudolf Tippelt, 268-284. Weinheim und Basel: Beltz Juventa.

Hirschberg, Marianne. 2010. »Das Recht auf Inklusive Bildung.«In Inklusive Bildung. Die UN-Konven- 
tion und ihre Folgen, herausgegben von Rolf Wernstedt, Marei John-Ohnesorg, 21-25. Berlin: Friedrich-Ebert-Stiftung.

Hirschberg, Marianne, und Christian Lindmeier. 2013. »Der Begriff ıInklusion< - Ein Grundsatz der Menschenrechte und seine Bedeutung für die Erwachsenenbildung.« In Zugänge zu Inklusion. Erwachsenenbildung, Behindertenpädagogik und Soziologie im Dialog, herausgegeben von Reinhard Burtscher, Eduard Jan Ditschek, Karl-Ernst Ackermann, Monika Kil und Martin Kronauer, 39-52. Bielefeld: wbv.

Holzkamp, Klaus. 1995. Lernen. Subjektwissenschaftliche Grundlegung. Studienausgabe. Frankfurt/M., New York: Campus.

Holzkamp, Klaus. 2004. „Wider den Lehr-Lern-Kurzschluß. Interview zum Thema `Lernen<. In Expansives Lernen, herausgegeben von Peter Faulstich und Joachim Ludwig, 29-38. Baltmannsweiler: Schneider Hohengehren.

Huinink, Johannes, und Torsten Schröder. 2014. Sozialstruktur Deutschlands. 2. Auflage. Stuttgart, Konstanz: UTB GmbH; UVK.

Koschek, Stefan, Meike Weiland, und Eduard Jan Ditschek. 2013. wbmonitor Umfrage 2012: Klima und Strukturen der Weiterbildungslandschaft. Zentrale Ergebnisse im Überblick. Bonn: Bundesinstitut für Berufsbildung.

Kroworsch, Susann. 2017. Das Recht auf inklusive Bildung. Allgemeine Bemerkung Nr. 4 des UNAusschusses für die Rechte von Menschen mit Behinderungen. Berlin: Deutsches Institut für Menschenrechte, Monitoring-Stelle UN-Behindertenrechtskonvention.

Kühn, Thomas, und Kay-Volker Koschel. 2018. Gruppendiskussionen. Ein Praxis-Handbuch. 2. Auflage. Wiesbaden: Springer VS.

Lindmeier, Bettina, und Christian Lindmeier. 2012. Pädagogik bei Behinderung und Benachteiligung. Band I: Grundlagen. Stuttgart: W. Kohlhammer.

Lindmeier, Christian, und Hans Weiß. 2017. »Pädagogische Professionalität im Spannungsfeld von sonderpädagogischer Förderung und inklusiver Erziehung und Bildung.« In Pädagogische Professionalität im Spannungsfeld von sonderpädagogischer Förderung und inklusiver Bildung, herausgegeben von Christian Lindmeier und Hans Weiß, 3-6. Weinheim: Beltz Juventa.

Loos, Peter, und Burkhard Schäffer. 2001. Das Gruppendiskussionsverfahren. Theoretische Grundlagen und empirische Anwendung. Opladen: Leske + Budrich.

Löser, Jessica; Werning, Rolf. 2015. »Inklusion - allgegenwärtig, kontrovers, diffus?« Erziehungswissenschaft, Nr. 51: 17-24.

Martin, Andreas, Stefanie Lencer, Josef Schrader, Stefan Koscheck, Hana Ohly, Rolf Dobischat et al. 2016. Das Personal in der Weiterbildung. Arbeits- und Beschäftigungsbedingungen, Qualifikationen, Einstellungen zu Arbeit und Beruf. Bielefeld: wbv.

Meisel, Klaus. 2012. »Bürde oder Paradigma? Inklusion in der Weiterbildung." In Inklusive Erwachsenenbildung. Kooperationen zwischen Einrichtungen der Erwachsenenbildung und der Behindertenhilfe, herausgegeben von Karl-Ernst Ackermann, Reinhard Burtscher, Eduard Jan Ditschek und Werner Schlummer, 17-25. Berlin: Eigenverlag Ges. Erwachsenenbild. u. Behinderung.

Papadopoulos, Christian. 2012. »Barrierefreiheit als didaktische Herausforderung." DIE Zeitschrift für Erwachsenenbildung, Nr. 2: 37-39.

Reichart, Elisabeth, Thomas Lux, und Hella Huntemann. 2018. Volkshochschul-Statistik. 56. Folge, Arbeitsjahr 2017. Bielefeld: wbv.

Schepers, Claudia. 2014. Wenn Kursleitende lernen. Orientierungssuche im Rahmen einer individuellen Professionalitätsentwicklung. Dissertation. Münster: Waxmann.

Sturm, Tanja. 2015. »Inklusion: Kritik und Herausforderung des schulischen Leistungsprinzips.« Erziehungswissenschaft, Nr. 51: 25-32. 
Wansing, Gudrun. 2015. »Was bedeutet Inklusion? Annäherung an einen vielschichtigen Begriff.» In Handbuch Behindertenrechtskonvention. Teilhabe als Menschenrecht - Inklusion als gesellschaftliche Aufgabe, herausgegeben von Theresia Degener und Elke Diehl, 43-51. Bonn: bpb Bundeszentrale für politische Bildung (Schriftenreihe/Bundeszentrale für Politische Bildung, 1506).

Witzel, Andreas. 2000. »Das problemzentrierte Interview.« Forum Qualitative Sozialforschung, Nr. 1: Art. 22.

\section{Der Autor}

Helge Stobrawe, M. A. (Soziologie und Sozialforschung), ist wissenschaftlicher Mitarbeiter an der Hochschule Bremen, Fakultät Gesellschaftswissenschaften, und arbeitet im Projekt INAZ.

Kontakt: helge.stobrawe@hs-bremen.de 